\title{
Knockdown of ISOC1 suppresses cell proliferation in pancreatic cancer in vitro
}

\author{
LI CHENG $^{1}$, YAN ZHAO ${ }^{2}$, MAOCHUN TANG $^{2}$, ZHENGSHENG LUO $^{1}$ and XINGPENG WANG ${ }^{1}$ \\ ${ }^{1}$ Department of Gastroenterology, Shanghai General Hospital of Nanjing Medical University, Shanghai 200080; \\ ${ }^{2}$ Department of Gastroenterology, School of Medicine, Shanghai Tenth People's Hospital, Tongji University, \\ Shanghai 200072, P.R. China
}

Received February 15, 2018; Accepted January 10, 2019

DOI: $10.3892 / 01.2019 .10082$

\begin{abstract}
Pancreatic cancer is a deadly disease that is frequently associated with mortality at the time of diagnosis due to rapid metastasis, which makes it unsuitable for operative surgery, and resistant to chemotherapy and radiation therapy. Isochorismatase domain-containing protein 1 (ISOC1) has putative isochorismatase activity, and is positively regulated by estrogen in human breast cancer. However, its role in pancreatic cancer has yet to be fully elucidated. Analysis from datasets downloaded from The Cancer Genome Atlas and Genotype-Tissue Expression databases indicated that the ISOC1 mRNA expression level was increased in pancreatic cancer tissues, compared with normal pancreatic tissues. In the present study, it was determined that the human pancreatic cancer cell lines SW 1990, PANC-1 and AsPC-1 had increased expression levels of ISOC1 mRNA, compared with human pancreatic ductal epithelial cells. Additionally, two of the pancreatic cancer cell lines, SW 1990 and PANC-1, transfected with lentivirus-delivered short hairpin RNA, to knockdown the expression of ISOC1, were established. Cell counting and MTT assays indicated that knockdown of ISOC1 decreased the ability of cell growth and proliferation in pancreatic cancer cells. Furthermore, Annexin V staining and caspase-3/7 activity assays demonstrated that inhibition of ISOC1 promoted cell apoptosis via elevation of the expression of caspase-3/7. Furthermore, inhibition of ISOC1 impaired the cell migration and invasive capability of the cells. In conclusion, ISOC1 exerts a role in pancreatic cancer cell growth and apoptosis, and may have a role in pancreatic cancer tumorigenesis.
\end{abstract}

\section{Introduction}

Pancreatic cancer is one of the most lethal cancer types, with a 5-year survival rate of $8 \%$ according to American Cancer

Correspondence to: Dr Xingpeng Wang, Department of Gastroenterology, Shanghai General Hospital of Nanjing Medical University, 100 Haining Road, Shanghai 200080, P.R. China

E-mail: richardwangxp@163.com

Key words: isochorismatase domain-containing protein 1, pancreatic cancer, cell proliferation, apoptosis, cell migration
Society Statistics in 2017 (1). The median overall survival time of patient with pancreatic cancer is only 5.0-7.2 months, according to statistics from France published in 2011 (2). The prognosis for patients with pancreatic cancer is poor due to this cancer type frequently being diagnosed at an advanced stage due to rapid progression, with limited symptoms at early stages meaning surgery is applicable only to a minority of patients $(<20 \%)$, but also because it is resistant to chemotherapeutic agents and radiation therapy (3). The current first-line chemotherapeutic drug, gemcitabine, for pancreatic cancer treatment remains unsatisfactory (2). Therefore, there is an urgent requirement for novel therapeutic approaches and drug combinations to provide a beneficial treatment for patients with pancreatic cancer.

Isochorismatase domain-containing protein 1 (ISOC1) may have putative isochorismatase activity, catalyzing the conversion of isochorismate into 2,3-dihydroxy-2,3-dihydrobenzoate and pyruvate via hydrolysis (4). Aside from its putative isochorismatase activity, the precise function of ISOC1 in human pancreatic cancer has not been fully elucidated. A number of recently published papers, using high-throughput techniques, including genetic sequencing and proteomic mass spectrometry, revealed that ISOC1 was one novel target that is positively regulated by estrogen in breast cancer, negatively regulated by microRNA-130a in neutrophil development and is a new peroxisomal constituent in human liver peroxisomes (5-8). Collectively, these recent observations indicate that ISOC1 may have important functions in ontogenesis, and that it is worthy of an in-depth investigation.

In the present study, the pancreatic cancer cell lines, SW 1990 and PANC-1, were used to investigate the potential role of ISOC1 in pancreatic cancer. ISOC1 expression was inhibited with short hairpin (sh)ISOC1 in order to determine its effects on cell growth, proliferation, apoptosis, migration and invasion following ISOC1 knockdown. The observations of the present study helped to improve our understanding of the role of ISOC1 in pancreatic cancer.

\section{Materials and methods}

, Reagents. The Rabbit polyclonal ISOC1 antibody anti-human ISOC1 was purchased from Abcam (\#ab118245; Cambridge, UK). Anti-GAPDH (\#sc-32233), peroxidase-conjugated 
antirabbit IgG (\#sc-2004) and anti-mouse IgG (\#sc-2005) were purchased from Santa-Cruz Biotechnology, Inc. (Dallas, TX, USA). RPMI 1640 medium with L-Gultamine (\#11875-093), fetal bovine serum (FBS; \#10100-147) and Lipofectamine ${ }^{\circledR}$ 2000 (\#11668019) were purchased from Thermo Fisher Scientific, Inc. (Waltham, MA, USA). Penicillin-streptomycin (\#1107440001) and Giemsa staining solution (\#32884) were purchased from Sigma-Aldrich; Merck KGaA (Darmstadt, Germany).

Cell lines and cell culture. Human pancreatic carcinoma SW 1990, PANC-1 and AsPC-1 cells and normal pancreatic duct epithelial (HPDE) cells were purchased from American Type Culture Collection (Manassas, VA, USA). These cell lines were maintained at $37^{\circ} \mathrm{C}$ in RPMI-1640 medium supplemented with $10 \%(\mathrm{v} / \mathrm{v})$ heat-inactivated FBS, penicillin (100 IU/ml) and streptomycin $(100 \mu \mathrm{g} / \mathrm{ml})$.

Construction of ISOCl knockdown lentivirus. According to the sequence of ISOC1 (NM_016048), shISOC1 and control shRNA (shCtrl) were designed. The sequences are as follows: shISOC1: 5'-CCGGCCATTTGAGTACCAGCATTTACT CGAGTAAATGCTGGTACTCAAATGGTTTTT-3'; and shCtrl: 5'-CCGGCCTTCTCCGAACGTGTCACGTCTCGA GTAAATGCTGGTACTCAAATGGTTTTT-3'. The two shRNAs $(100 \mathrm{ng} / \mu \mathrm{l})$ were inserted into the plasmid GV115 (U6-MCS-CMV-EGFP; Shanghai GeneChem Co., Ltd., Shanghai, China). Recombinant lentiviruses were generated by transfection of 293T cells (Cell Bank of Chinese Academy of Science, Shanghai, China), in a $10 \mathrm{~cm}$ dish at $80 \%$ confluency, with $20 \mu \mathrm{g}$ GV115 plasmid and the helper plasmids, including $15 \mu \mathrm{g}$ Helper 1.0 and $15 \mu \mathrm{g}$ Helper 2.0 (Shanghai GeneChem Co.,Ltd.) using Lipofectamine ${ }^{\circledR} 2000$. After 48 h of transfection, viral supernatants were collected, centrifuged at $10,000 \times \mathrm{g}$ at $4^{\circ} \mathrm{C}$ to remove cell debris and then filtered through $0.45 \mu \mathrm{m}$ polyvinylidene fluoride membranes. Lentivirus titer (TU/ml) was measured using HIV p24 antigen ELISA kit 2.0 (\#0801002; ZeptoMetrix, Buffalo, NY, USA). SW 1990 and PANC-1 cells were infected with shISOC1 lentivirus or control virus at multiplicity of infection of 2 using polybrene (\#H9268; Sigma-Aldrich; Merck KGaA). At 72 h after infection, green fluorescent protein (GFP) expression was observed and the achieved infection efficiency was $80 \%$, and the expression of ISOC1 was analyzed by reverse transcription-quantitative polymerase chain reaction (RT-qPCR) and western blotting, according to the subsequent protocols.

MTT assay. To test directly whether ISOC1 inhibition would affect cell viability and proliferation, an MTT assay was performed to compare the effects of decreased ISOC1 expression on cell numbers. SW 1990 and PANC-1 cells were transfected with shISOC1 lentivirus or control virus at a multiplicity of infection of 2 using polybrene. Stably-transfected cells were seeded into a 96 -well plate $\left(2 \times 10^{3}\right.$ cells/well $)$ at $37^{\circ} \mathrm{C}$ for $16 \mathrm{~h}$, and after cell adhesion, $20 \mu \mathrm{l} \mathrm{MTT}(5 \mathrm{mg} / \mathrm{ml}$; Genview, Houston, TX, USA; \#JT343) was added and cultured at $37^{\circ} \mathrm{C}$ for $4 \mathrm{~h}$. Subsequently, the media was completely removed and $100 \mu \mathrm{l}$ dimethyl sulfoxide was added and optical density 490 was determined on a microplate reader. The viability of the cells was assessed by measuring the absorbance at $490 \mathrm{~nm}$ using a Microplate Absorbance Reader (Tecan Group, Ltd., Männedorf, Switzerland; \#M2009PR). Each experiment was repeated three times.

Western blot analysis. Total protein was extracted from cell lines by incubating with 2X Lysis Buffer [100 mM Tris- $\mathrm{HCl}$ (pH 6.8), 4\% SDS, 20\% glycerol and 2\% mercaptoethanol] on ice for $10 \mathrm{~min}$ and sonication $5 \mathrm{sec}$ each time for 4 times (200 W with 2 sec interval). Total protein was measured through the bicinchoninic acid method and separated by $10 \%$ SDS-PAGE. A total of $30 \mu \mathrm{g}$ proteins were run on gels and transferred to polyvinyl difluoride membranes. The membranes were blocked in 5\% bovine serum albumin (Gibco; Thermo Fisher Scientific, Inc.; \#37525) at room temperature for 1 hand then probed with the primary antibodies overnight at $4^{\circ} \mathrm{C}$. The membranes were incubated with appropriate secondary antibodies (1:2,000 dilution), either peroxidase-conjugated anti-mouse $\mathrm{IgG}$ or anti-rabbit IgG at room temperature for $1 \mathrm{~h}$. GAPDH was used as a loading control. Subsequently, the antigen-antibody complexes were detected by using an enhanced chemiluminescent blotting analysis system.

$R T-q P C R$. Total RNA was extracted from cells using a SuperfecTRI, Total RNA Isolation reagent (Shanghai Pufei Biotechnology Co., Ltd., Shanghai, China; \#3101-100). cDNA was transcribed from $1 \mu \mathrm{g}$ total RNA using a Promega M-MLV kit (Promega Corporation, Madison, WI, USA; \#M1705), according to the manufacturer's protocol. cDNA was used as the template for qPCR detection with primers and SYBR ${ }^{\circledR}$ Master Mixture on LightCycler480 (Roche Diagnostics, Basel, Switzerland). The primers for ISOC1 are forward, 5'-CGACAT GCACCGCAAATTCG-3', and reverse, 5'-TGAGCTGGATCT GCAACGG-3'. The primers for GAPDH are forward, 5'-TGA CTTCAACAGCGACACCCA-3', and reverse, 5'-CACCCTGTT GCTGTAGCCAAA-3'. The qPCR was performed with the following conditions: i) Hold at $95^{\circ} \mathrm{C}$ for $30 \mathrm{sec}$, ii) step-PCR for 40 cycles of $95^{\circ} \mathrm{C}$ for $5 \mathrm{sec}$ and $60^{\circ} \mathrm{C}$ for $30 \mathrm{sec}$; and iii) dissociation at $95^{\circ} \mathrm{C}$ for $15 \mathrm{sec}, 60^{\circ} \mathrm{C}$ for $30 \mathrm{sec}$ and $95^{\circ} \mathrm{C}$ for $15 \mathrm{sec}$. The expression of the ISOC 1 gene, and endogenous control gene GAPDH, was detected and analyzed. The relative mRNA expression level of ISOC1 in each sample was calculated using the comparative expression level $2^{-\Delta \Delta \mathrm{Cq}}$ method (9). All experiments were conducted in triplicate for each data point.

ISOC1 mRNA level analysis from The Cancer Genome Atlas (TCGA) and Genotype-Tissue Expression (GTEx) Portal. RNA sequencing results of pancreatic adenocarcinoma were downloaded from TCGA (http://www.cbioportal.org/datasets). RNA sequencing results of normal pancreas tissue were downloaded from GTEx (phs000424.v6.p1; ftp://ftp.ncbi.nlm.nih. gov/dbgap/studies/phs000424). ISOCl sequencing results were extracted, normalized and integrated as transcripts per million.

Matrigel invasion assay. The Matrigel invasion assay was performed in a 24-well plate Transwell system (Corning Inc. Corning; NY, USA; \#07-200-537). The Transwell inserts were coated with $100 \mu \mathrm{l}$ Matrigel and incubated at $37^{\circ} \mathrm{C}$ for $30 \mathrm{~min}$. A total of $5 \times 10^{3}$ SW 1990 cells were harvested and resuspended in $100 \mu \mathrm{l}$ RPMI-1640 medium to the upper chamber of the Transwell system. The lower chamber was infused with 
A
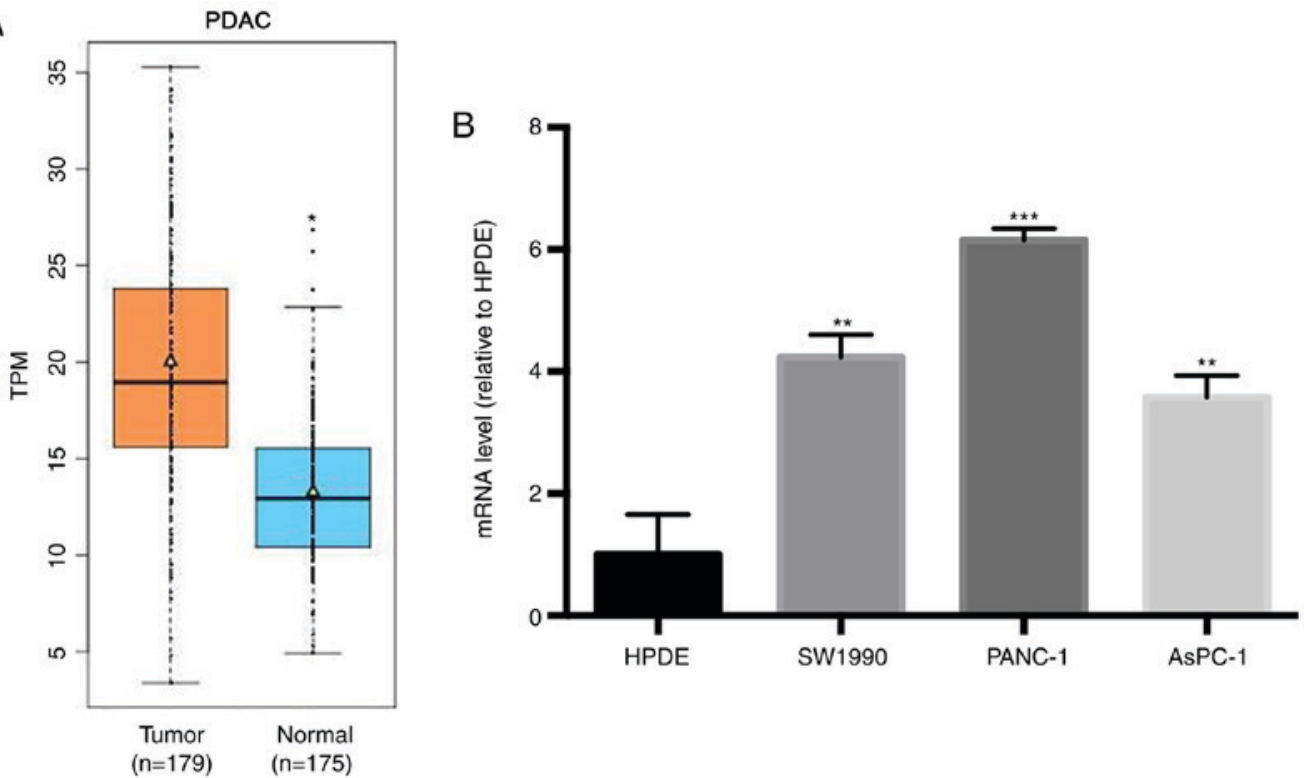

Figure 1. ISOC1 is expressed in human PDAC tissues and cell lines. (A) mRNA level of ISOC1 in human PDAC tissues (n=179) and the adjacent normal pancreas tissues $(n=175)$. The triangle represents the mean gene expression values. (B) Reverse transcription-quantitative polymerase chain reaction analysis of ISOC1 knockdown efficiency in HPDE, SW 1990, PANC-1 and AsPC-1 cells. ${ }^{*} \mathrm{P}<0.05$, compared with PDAC tissues. ${ }^{* *} \mathrm{P}<0.01$ and ${ }^{* * * *} \mathrm{P}<0.001$, compared with HPDE. PDAC, pancreatic adenocarcinoma; TPM, transcripts per million; ISOC1, isochorismatase domain-containing protein 1.

$100 \mu 1$ RPMI-1640 with $30 \%$ FBS. The Transwell system was incubated at $37^{\circ} \mathrm{C}$ for $20 \mathrm{~h}$, and then the gel and cells in the upper chamber were cleared. Following $40 \%$ formalin fixation at room temperature for $15 \mathrm{~min}$, the membrane was stained with Giemsa staining solution for 3-5 min at room temperature. Phase contrast images were captured and the cells on the lower side of the membrane were counted in 6 random visual fields under a x20 objective lens of an inverted microscope (CKX41, Olympus Corporation, Tokyo, Japan).

Celigo counting assay. SW 1990 and PANC-1 cells were transfected with shISOC1 lentivirus or control virus at a multiplicity of infection of 2 using polybrene. A total of $15 \times 10^{2}$ cells/well were seeded in RPMI-1640 medium in a 96 well plate. The GFP-expressing cell number was counted with a Celigo image cytometer once a day at $37^{\circ} \mathrm{C}$ (Nexcelom Bioscience, Lawrence, MA, USA) for 5 days.

Annexin V assay. Apoptosis of SW 1990 cells was detected using an Annexin V-APC staining kit (eBioscience; Thermo Fisher Scientific, Inc.; \#88-8007) after lentivirus infection at multiplicity of infection of 2 using polybrene for 6 and 8 days. Cells $\left(5 \times 10^{5}\right.$ cells/dish) were cultured at $37^{\circ} \mathrm{C}$ in $10-\mathrm{cm}$ dishes to reach $80 \%$ confluency, and then they were harvested, washed twice with PBS and stained with $10 \mu \mathrm{l}$ Annexin V-APC at $37^{\circ} \mathrm{C}$ for $10-15 \mathrm{~min}$. Subsequently, cells were kept on ice in the dark and subjected to apoptosis analysis with a flow cytometer (EMD Millipore; \#Guava easyCyte HT; Billerica, MA, USA). Data were analyzed with Guava Suite 3.3 software (EMD Millipore).

Caspase-Glo 3/7 Assay. SW 1990 and PANC-1 cells were transfected with shISOC1 lentivirus or control virus at a multiplicity of infection of 2 using polybrene. Caspase 3/7 activity in SW 1990 and PANC-1 cells following shCtrl or
shISOC1 treatment was detected using a Caspase-Glo 3/7 kit (Promega Corporation). A total of $1 \times 10^{4}$ cells infected with shCtrl or shISOC1 were seeded in 96-well plates. After 3 days growth at $37^{\circ} \mathrm{C}, 100 \mathrm{ml}$ Caspase $3 / 7$ reagent were added to each well, mixed and incubated for $1 \mathrm{~h}$ at room temperature. Luminescence was measured using a M2000 Infinite Pro instrument (Tecan Group, Ltd.). Results of Caspase 3/7 activity were expressed as percentage of the negative control.

Migration assay. A total of $3 \times 10^{4}$ cells were seeded in 96 well plate and incubated at $37^{\circ} \mathrm{C}$ for $16 \mathrm{~h}$ to achieve $90 \%$ confluency. A 96 wounding replicator (VP scientific, Inc.; \#VP408FH; San Diego, CA, USA) was used to scratch a monolayer across the surface of the well. Subsequently, the well was gently washed with RPMI-1640 medium to remove the detached cells. The cells were then incubated with fresh RPMI-1640 medium (containing $0.5 \% \mathrm{FBS}$ ) at $37^{\circ} \mathrm{C}$ for $24 \mathrm{~h}$. Photos were captured at 0,8 and $24 \mathrm{~h}$. The wound area was analyzed and measured with Image J 1.51 software (National Institutes of Health, Bethesda, MD, USA).

Statistical analysis. Data are expressed as mean \pm standard deviation from three independent experiments. The statistical analyses were performed using the GraphPad Prism (Version 6.0; GraphPad Software, Inc., La Jolla, CA, USA). Student's t-test was used for comparison between two groups. Two-way ANOVA with Bonferroni post-hoc test were used to compare the differences among multiple groups. $\mathrm{P}<0.05$ was considered to indicate a statistically significant difference.

\section{Results}

ISOC1 is expressed in human pancreatic adenocarcinoma tissues and cell lines. The expression levels of ISOC1 mRNA 

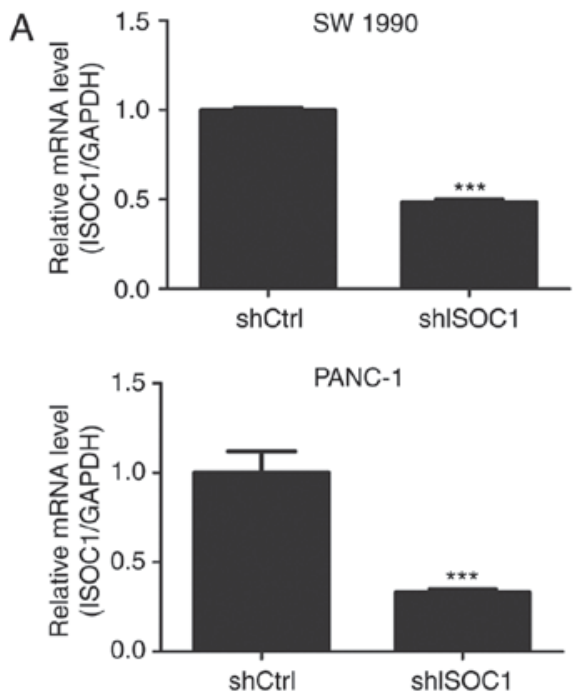

B

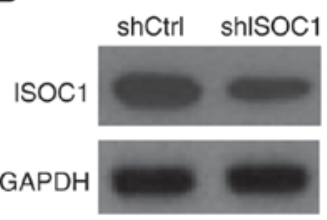

PANC-1

Figure 2. Knockdown efficiency of ISOC1 by lentivirus infection in the pancreatic cell lines SW 1990 and PANC-1. (A) Reverse transcription-quantitative polymerase chain reaction analysis of ISOC1 knockdown efficiency in SW 1990 cells. (B) Western analysis validated the knockdown efficiency of ISOC1 in SW 1990 cells. ${ }^{* * *} \mathrm{P}<0.01$, compared with the shCtrl group. ISOC1, isochorismatase domain-containing protein 1 ; shCtrl, control short hairpin RNA.

in 179 pancreatic adenocarcinoma (PDAC) tissues and 4 normal pancreatic tissues were downloaded from TCGA database. The expression levels of ISOC1 mRNA from a further 171 normal pancreatic tissues were downloaded from the GTEx portal database. The mRNA expression levels of ISOC1 from PDAC and normal pancreatic tissues is plotted in the boxplot format in Fig. 1A. As depicted in Fig. 1A, the level of ISOC1 mRNA in PDAC tissues was significantly increased, compared with normal pancreatic tissues. Subsequently, the expression level of ISOC1 mRNA was assessed in the human PDAC cell lines, SW 1990, PANC-1 and AsPC-1, and in the normal cell line, HPED. RT-PCR analysis revealed that ISOC1 mRNA was expressed in all three human PDAC cell lines (Fig. 1B). Furthermore, the ISOC1 mRNA levels in PDAC cells were significantly increased, compared with normal cells $(\mathrm{P}<0.01)$. Therefore, elevated expression levels of ISOC1 may exert an important role in the pathogenesis of human PDAC.

Successful knockdown of ISOC1 in SW 1990 and PANC-1. To investigate the function of ISOC1 in pancreatic cancer, the expression of ISOC1 was inhibited by infection with shISOC1 in the SW 1990 and PANC-1 PDAC cell lines. The inhibition efficiency was confirmed by examining the mRNA level of ISOC1 using RT-qPCR, and the protein expression level of ISOC1 by western blotting, in shISOC1- and shCtrl-treated cell lines. The majority of the cells revealed GFPpositive expression, indicating a high infection efficiency. Furthermore, the mRNA levels of ISOC1 were significantly downregulated in shISOC1-treated cells, compared with the shCtrl group (Fig. 2A). Consistently, treatment with shISOC1 decreased the protein level of ISOC1 in the SW 1990 and PANC-1 cell lines (Fig. 2B). Collectively, these observations confirmed that ISOC1 silencing in SW 1990 and PANC-1 cell lines had been successfully accomplished.

Inhibition of cell proliferation by shISOCl. The potential effects of ISOC1 on cell proliferation were examined in SW 1990 and PANC-1 cells by Celigo cell counting and MTT assays. The same numbers of SW 1990 and PANC-1 cells (1,500 cells/well) from the shISCO1 and shCtrl groups were seeded into 96-well plates, and cell numbers were continuously counted using a Celigo plate reader over the course of the following 5 days. Cellular images and the cell number plots are depicted in Fig. 3A and B, respectively. The results indicated that SW 1990 and PANC-1 cells infected with shISOC1 presented a significantly reduced proliferative rate, compared with the control group (Fig. 3B and C). The MTT assay revealed similar results (Fig. 3D and E). During the 5 days of growth, the proliferation rates of the SW 1990 and PANC-1 cells were significantly inhibited by ISOC1 knockdown, compared with the control group. The aforementioned results revealed that ISOC1 may serve an important role in pancreatic cancer cell proliferation.

Knockdown of ISOCl induces cell apoptosis. To determine the possible underlying mechanism of inhibition of cell proliferation, apoptosis was analyzed using Annexin V-staining flow cytometry and caspase $3 / 7$ assay. As depicted in Fig. 4A and $\mathrm{B}$, the percentages of apoptotic cells in shISOC1-infected SW 1990 and PANC1 cells were significantly increased, compared with that in the shCtrl group. In agreement with the flow cytometry data, caspase 3/7 activity was significantly increased in shISOC1-infected cells, compared with that in the shCtrl group (Fig. 4C). These results indicated that knockdown of ISOC1 inhibited cell proliferation through induction of cell apoptosis.

Knockdown of ISOC1 impairs cell migration and invasion ability. In addition to the inhibition of cell proliferation by ISOC1 knockdown, the effects of cell migration (up to $24 \mathrm{~h}$ ) and invasion $(16 \mathrm{~h})$ were also investigated in a shorter observational window, compared with the MTT assay (5 days). The same numbers of SW 1990 and PANC-1 cells (3x10 $\left.{ }^{4}\right)$ infected with shISOC1 and shCtrl were used for both assays. In the migration assay, significantly reduced migration rates were identified in the shISOC1 group, compared with the shCtrl group at $24 \mathrm{~h}(\mathrm{P}<0.01$; Fig. 5A and $\mathrm{B})$. Consistently, significantly fewer cells invaded into the lower side of the chamber in the shISOC1 group, compared with the shCtrl group (Fig. 5C and D) after $16 \mathrm{~h}$ of incubation. Collectively, these results indicated that ISCO1 inhibition impaired the migration and invasive abilities of the pancreatic cells.

\section{Discussion}

In the present study, it was determined that the ISOC1 mRNA level is significantly increased in PDAC tissues, compared with normal pancreatic tissues, and the same trend was observed 
A
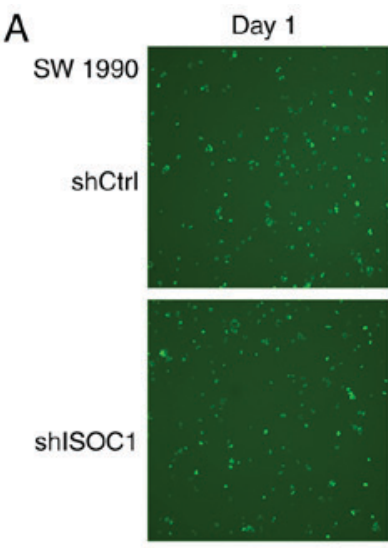

PANC-1

shCtrl

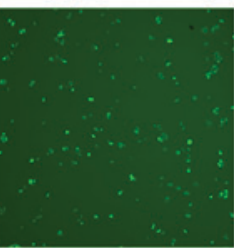

shISOC1

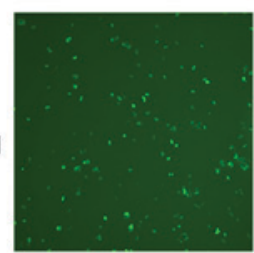

\section{B}
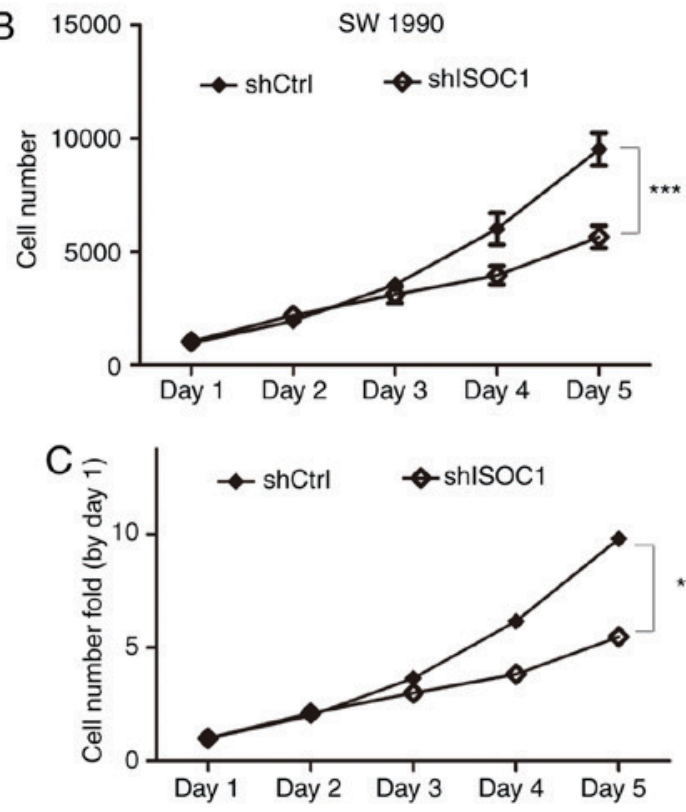

D
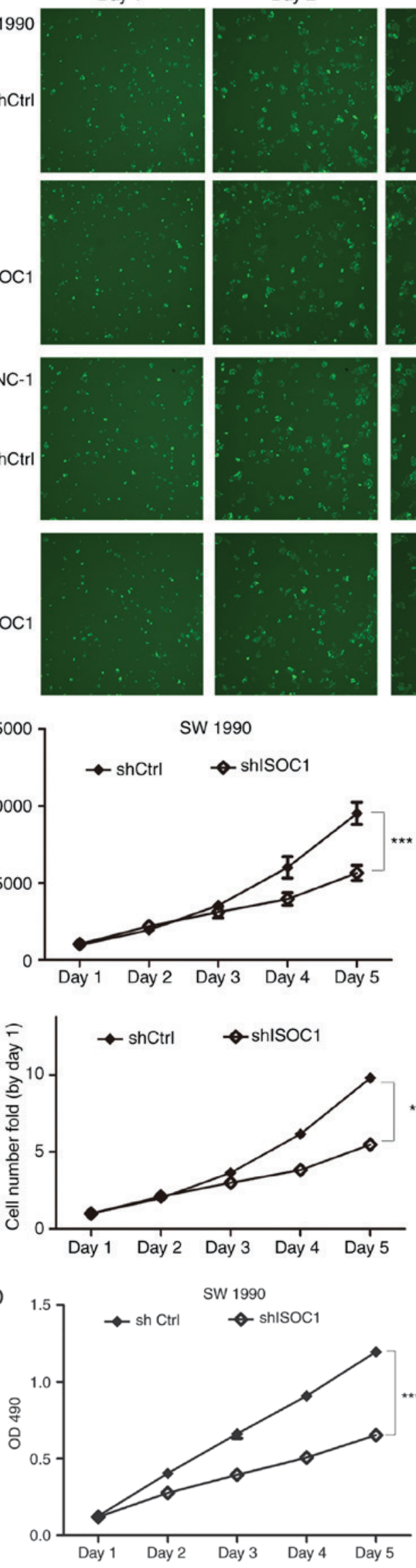

SW 1990

Day 3 

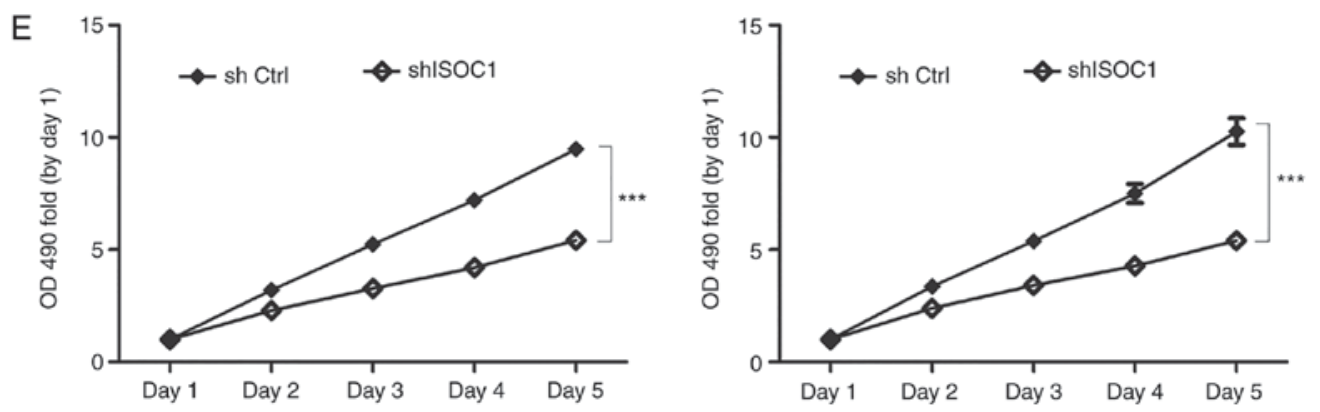

Figure 3. Continued. (E) Curve of OD490 fold of SW 1990 and PANC-1 cells following ISOC1 knockdown. Scale bars, $100 \mu$ m. ${ }^{* * * *} \mathrm{P}<0.001$, comparing the shCtrl and shISOC1 groups. ISOC1, isochorismatase domain-containing protein 1; shCtrl, control short hairpin RNA; OD, optical density.

A

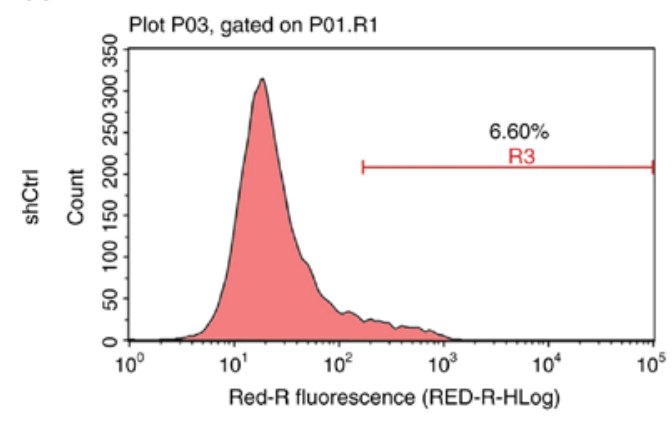

ANNEXIN-V

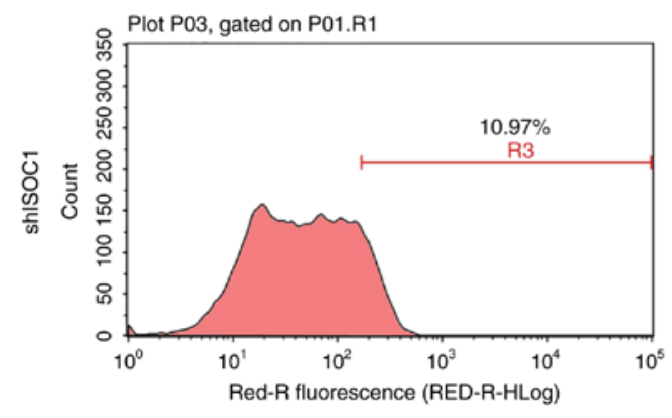

ANNEXIN-V

B

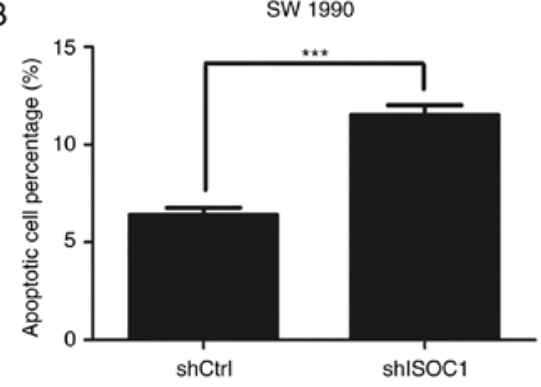

C

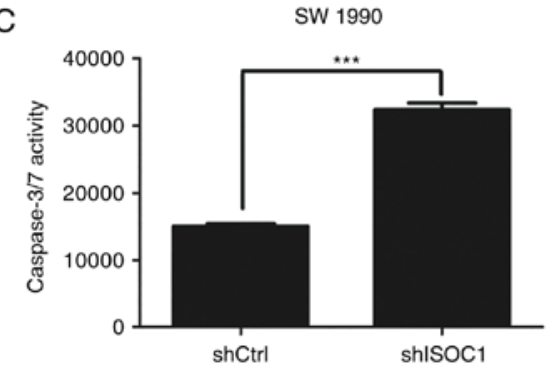

PANC-1
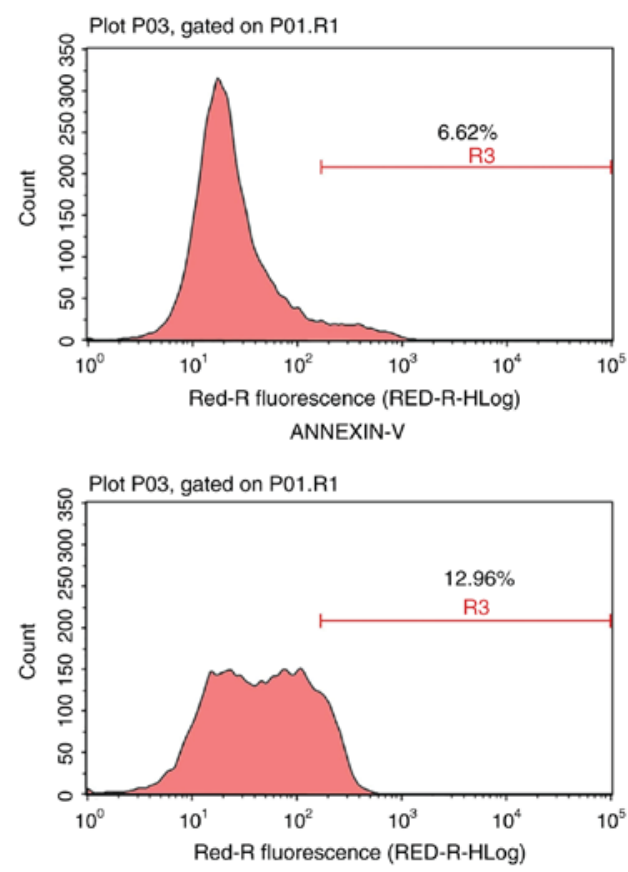

ANNEXIN-V

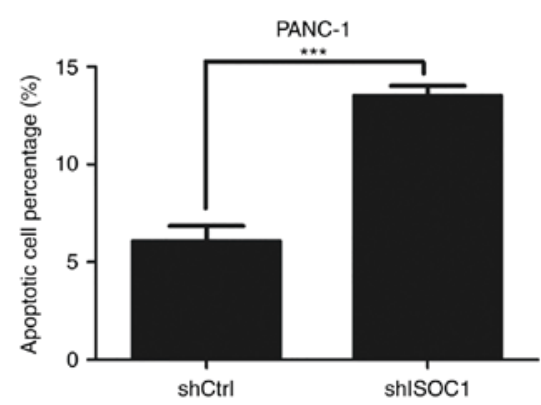

PANC-1

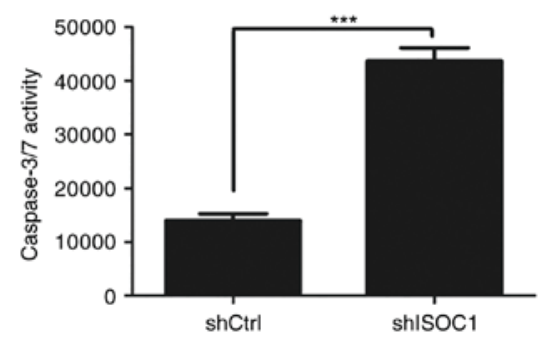

Figure 4. Depletion of ISOC1 promotes cell apoptosis. (A) Flow cytometry combined with Annexin V staining was used to analyze cell apoptosis in SW 1990 and PANC-1 knockdown. (B) Statistical analysis of apoptotic cell in SW1990 and PANC-1 with and without ISOC1 depletion. (C) Caspase 3/7 assay was used to determine the caspase 3/7 activity following ISOC1 knockdown. ${ }^{* * *} \mathrm{P}<0.01$, compared with the shCtrl group. ISOC1, isochorismatase domain-containing protein 1; shCtrl, control short hairpin RNA. 
A
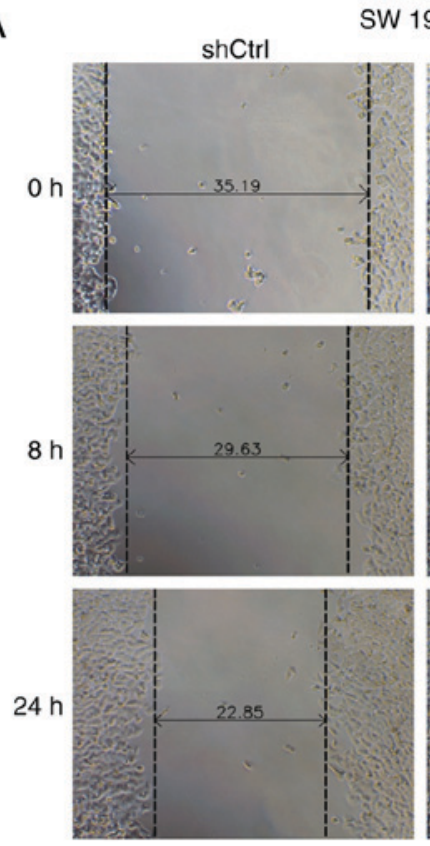

B

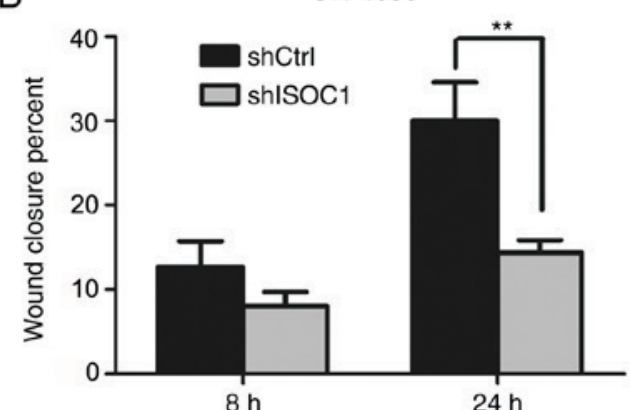

C

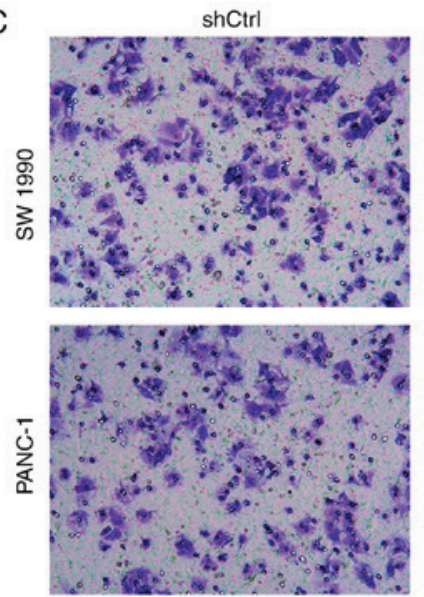

shISOc1
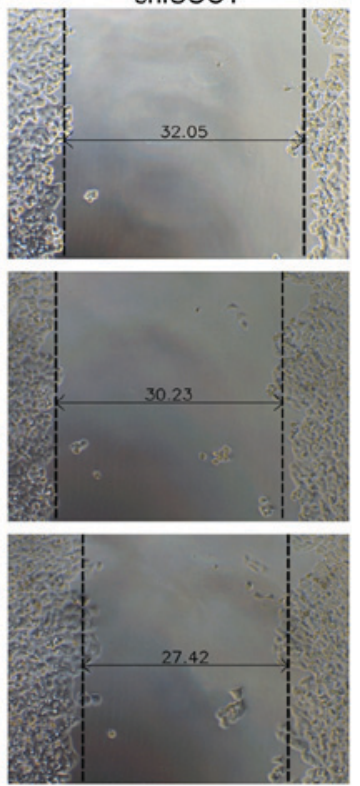

SW 1990
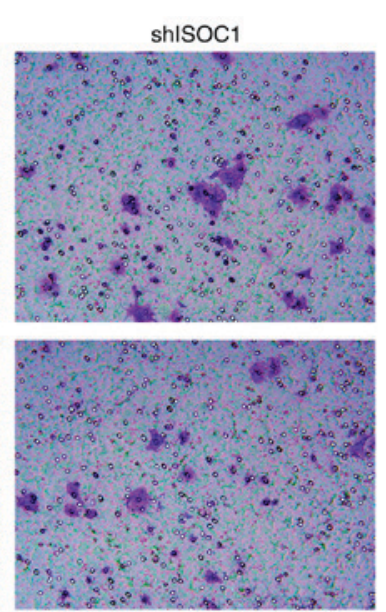
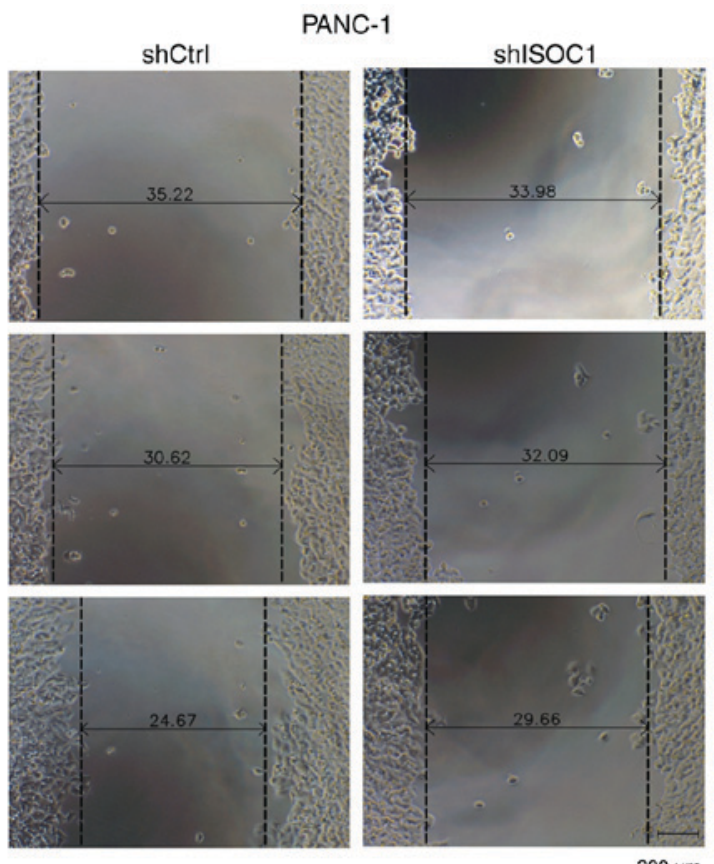

PANC-1

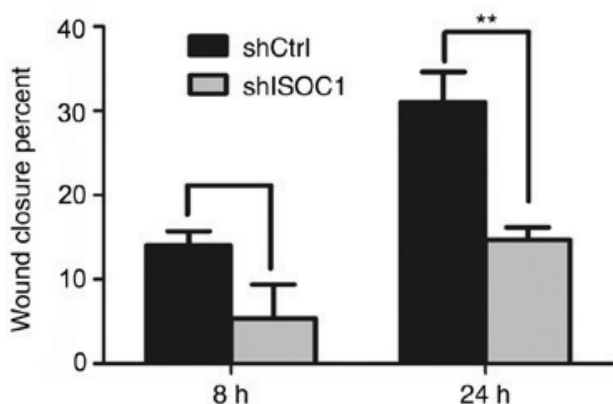

SW 1990

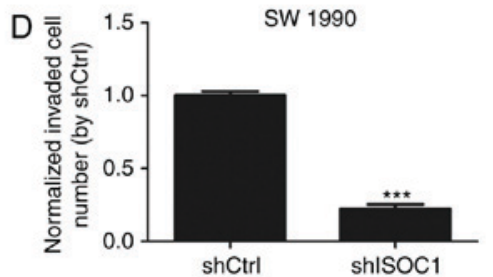

PANC-1

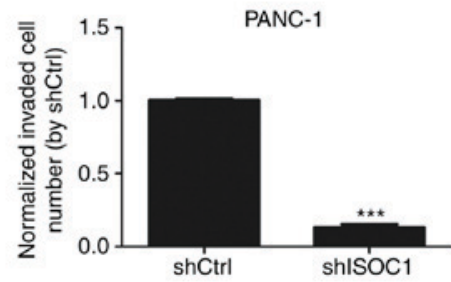

Figure 5. Knockdown of ISOC1 impairs cell migration and invasion ability. (A) Decrease in cell migration following ISOC1 inhibition by shRNA in SW 1990 cells. Scale bar=200 $\mu \mathrm{m}$. (B) Quantitative analysis of migration assay result. (C) Decrease in cell invasion ability in SW 1990 and PANC-1 after ISOC1 knockdown. Images were captured under a x20 objective lens. (D) Quantitative analysis of the number of cells that invaded the lower side of the membrane shISOC1 and shCtrl group. ${ }^{* *} \mathrm{P}<0.01$ and ${ }^{* * * *} \mathrm{P}<0.001$, compared with the shCtrl group. ISOC1, isochorismatase domain-containing protein 1; shCtrl, control short hairpin RNA.

in PDAC cell lines, compared with normal pancreatic cells. Furthermore, the shISOC1-infected PDAC cell lines, SW 1990 and PANC-1, were demonstrated to have a reduced level of ISOC1 expression, compared with the respective control cell lines. Cell growth and proliferation was indicated to be significantly inhibited following ISOC1 knockdown. Furthermore, this inhibition of the cell growth and proliferation was determined to be associated with cell apoptosis. Finally, ISOC1 inhibition also resulted in an impairment of cell migration and invasion. To the best of our knowledge, this is the first study to have revealed how ISOC1 is involved in pancreatic cancer growth and migration.

Pancreatic cancer is notoriously difficult to detect early and to cure. Current therapeutic options for patients are limited and unsatisfactory (2). The observations of the present study 
demonstrated that ISOC1 may be a new potential intervention target in pancreatic cancer. Currently, it has not been established whether the effects of knockdown of ISOC1 on pancreatic cancer cells are dependent on, or otherwise associated with, the putative isochorismatase enzyme activity of ISOC1. Recently reported potential isochorismatase inhibitors, including suberoylanilide hydroxamic acid (10) and cinnamic acid (11), may help to address the previous question. Notably, ISOC2, an ISOC1 homolog, was determined to directly interact with the tumor suppressor $\mathrm{p} 16^{\mathrm{INK} 4 \mathrm{a}}$, and to negatively regulate p16 ${ }^{\text {INK4a }}$ (12). Overexpressed ISOC2 inhibited the expression of p16 ${ }^{\text {INK4a }}$. Our hypothesis is that ISOC1 may perform an action on $\mathrm{p} 16^{\text {INK4a }}$ similar to that of ISOC2, and that this may be the mechanism utilized by ISCO1 in terms of its role in tumor progression.

Previous studies revealed that ISOC1 was regulated by estrogen in the breast cancer cell line, MCF-7 $(7,8)$. Furthermore, small interfering RNA knockdown of ISOC1 inhibits the growth of MCF-7 cells, which indicated that ISOC1 is involved in the growth of breast cancer cells (7). These results are consistent with those in the present study. This indicates that the positive regulation of ISOC1 on cancer cell proliferation may not be limited to pancreatic cancer and breast cancer, but may be a more widespread phenomenon in different types of cancer. It would be worthwhile to test the validity of this hypothesis. The previous study revealed that, in breast cancer, inhibition of ISOC1 results in a small decrease in the migration ability of the cells, although this difference was not observed to be significant (7). However, in the present study, it was determined that knockdown of ISOC1 did significantly decrease the migration and invasive ability of the pancreatic cancer cells. These different effects elicited by ISOC1 knockdown may be due to the different cancer context.

In conclusion, in the present study, the function of ISOC1 in pancreatic cancer was examined and it was determined that silencing ISOC1 expression induced apoptosis and suppressed cell proliferation in pancreatic cancer. These results indicated that ISOC1 could be a potential therapeutic target for the treatment of pancreatic cancer. However, further studies are required to fully elucidate the regulatory mechanisms of ISOC1 in pancreatic tumorigenesis.

\section{Acknowledgements}

Not applicable.

\section{Funding}

This study was supported by National Natural Science Foundation (grant no. 81300350) and by Science and Technology Commission Foundation of Shanghai Municipality (grant no. 16411950404).

\section{Availability of data and materials}

All data generated or analyzed during this study are included in this published article.

\section{Authors' contributions}

XW proposed the hypothesis and designed the study. LC performed the MTT and apoptosis assays, analyzed the data and wrote the article. YZ, MT and ZL performed the cell wound healing and Transwell assays, and analyzed the data.

\section{Ethics approval and consent to participate}

Not applicable.

\section{Patient consent for publication}

Not applicable.

\section{Competing interests}

The authors declare that they have no competing interests.

\section{References}

1. American Cancer Society: Cancer Facts \& Figures 2017. American Cancer Society, Atlantla, GA, 2017.

2. Conroy T, Desseigne F, Ychou M, Bouché O, Guimbaud R, Bécouarn Y, Adenis A, Raoul JL, Gourgou-Bourgade S, de la Fouchardière $\mathrm{C}$, et al: FOLFIRINOX versus gemcitabine for metastatic pancreatic cancer. N Eng J Med 364: 1817-1825, 2011.

3. Hidalgo M, Cascinu S, Kleeff J, Labianca R, Löhr JM, Neoptolemos J, Real FX, Van Laethem JL and Heinemann V: Addressing the challenges of pancreatic cancer: Future directions for improving outcomes. Pancreatology 15: 8-18, 2015.

4. Walsh CT, Liu J, Rusnak F and Sakaitani M: Molecular studies on enzymes in chorismate metabolism and the enterobactin biosynthetic-pathway. Chem Rev 90: 1105-1129, 1990.

5. Gronemeyer T, Wiese S, Ofman R, Bunse C, Pawlas M, Hayen H, Eisenacher M, Stephan C, Meyer HE, Waterham HR, et al: The proteome of human liver peroxisomes: Identification of five new peroxisomal constituents by a label-free quantitative proteomics survey. PLoS One 8: e57395, 2013.

6. Pedersen CC,Refsgaard JC,Ostergaard O,Jensen LJ,Heegaard NH, Borregaard N and Cowland JB: Impact of microRNA-130a on the neutrophil proteome. BMC Immunol 16: 70, 2015.

7. Yamaga R, Ikeda K, Boele J, Horie-Inoue K, Takayama K, Urano T, Kaida K, Carninci P, Kawai J, Hayashizaki Y, et al: Systemic identification of estrogen-regulated genes in breast cancer cells through cap analysis of gene expression mapping. Biochem Biophys Res Commun 447: 531-536, 2014.

8. Rezaul K, Thumar JK, Lundgren DH, Eng JK, Claffey KP, Wilson L and Han DK: Differential protein expression profiles in estrogen receptor-positive and -negative breast cancer tissues using label-free quantitative proteomics. Genes Cancer 1: 251-271, 2010.

9. Livak KJ and Schmittgen TD: Analysis of relative gene expression data using real-time quantitative PCR and the 2- $\Delta \Delta \mathrm{CT}$ method. Methods 25: 402-408, 2001.

10. Fischer JJ, Michaelis S, Schrey AK, Diehl A, Graebner OY, Ungewiss J, Horzowski S, Glinski M, Kroll F, Dreger M and Koester H: SAHA capture compound-A novel tool for the profiling of histone deacetylases and the identification of additional vorinostat binders. Proteomics 11: 4096-4104, 2011.

11. Hubrich F, Mordhorst S and Andexer JN: Cinnamic acid derivatives as inhibitors for chorismatases and isochorismatases. Bioorg Med Chem Lett 23: 1477-1481, 2013.

12. Huang XY, Shi ZC, Wang W, Bai J, Chen Z, Xu J, Zhang D and $\mathrm{Fu}$ S: Identification and characterization of a novel protein ISOC2 that interacts with p16(INK4a). Biochem Biophys Res Commun 361: 287-293, 2007. 